\title{
Actividad antimicrobiana de líquenes de la cuenca alta del rio Bogotá
}

\author{
Antimicrobial activity of lichens in the upper basin of Bogotá \\ river
}

Oscar E. Rodríguez A. ${ }^{1}$, William A. Andrade B. ${ }^{\text {, }}$ Fabio E. Díaz L. ${ }^{1}$, Bibiana Moncada ${ }^{2}$.

\section{Resumen}

Objetivo: Determinar la actividad antimicrobiana de extractos de diferentes polaridades de las especies de Everniastrum columbiense, Flavopunctelia flaventor, Parmotrema simulans, Ramalina celastri, Teloschistes exilis y Usnea sp., recolectadas en el margen occidental de la cuenca alta del Río Bogotá, a los $05^{\circ} 07^{\prime} 23,4$ ”N y 7339'10,5”W; frente a los microorganismos Staphylococcus aureus y Klebsiella pneumoniae. Materiales y métodos. Se utilizó el método de difusión en gel por perforación en placa. Resultados. Los extractos de Flavopunctelia flaventor, Everniastrum columbiense, Parmotrema simulans, Usnea Sp y Ramalina celastri, presentaron actividad antibacteriana frente a Staphylococcus aureus. Y los extractos de Flavopunctelia flaventor, Usnea Sp. y Ramalina celastri, presentaron actividad antibacteriana frente a Klebsiella pneumoniae.

Palabras clave: Líquenes, actividad antimicrobiana.

\begin{abstract}
Objetive: To determine the antimicrobial activity of extracts of different polarities species Everniastrum columbiense, flaventor Flavopunctelia, Parmotrema simulans, Ramalina celastri, Teloschistes exilis and Usnea sp, collected on the western margin of the basin alta of Río Bogotá, to $05 .^{\circ} 07$ '23.4 "N and $73^{\circ} 39^{\prime} 10.5^{\prime \prime} \mathrm{W}$; against microorganisms Staphylococcus aureus and Klebsiella pneumoniae. Materials and methods. Diffusion method was used in gel plate by drilling. Results: Extracts of Flavopunctelia flaventor, Everniastrum columbiense, Parmotrema simulans, Usnea Sp and Ramalina celastri showed antibacterial activity against Staphylococcus aureus with antibacterial. And extracts Flavopunctelia flaventor, Usnea Sp and Ramalina celastri showed antibacterial activity against Klebsiella pneumoniae.
\end{abstract}

Keywords: Lichens, antimicrobial activity

\footnotetext{
Grupo de Investigación en Productos Naturales y Contaminación Ambiental. Ingeniería Ambiental, Universidad El Bosque

2. Curadora Herbario Forestal UDBC-Sección Criptógamas, Grupo Colombiano de Liquenología GCOL, Universidad Distrital Francisco José de Caldas

Correspondencia: rodriguezoscare@unbosque.edu.co
} 


\section{Introducción}

Los líquenes son probablemente los más desconocidos y pobremente apreciados organismos en el mundo biológico (1). La "maldición" que sobre ellos colocó Lineo llamándolos "las pobres basuras de la vegetación”, aún hoy dos siglos después no ha sido levantada. Esta consideración sobre los líquenes es debida a que ellos se encuentran desordenadamente en cualquier parte, no son plantas semejantes a musgos y no se parecen a las especies de hongos conocidas hasta el momento (2). Según Filho los líquenes son vegetales complejos originados de una íntima interpenetración de hongos superiores o inferiores y algas casi siempre unicelulares (3), de modo que ambos forman nuevas unidades morfológicas, las cuales en la mayoría de las veces completamente diferente a sus componentes, o bien, los líquenes son asociaciones estables entre un fotobionte y un micobionte, capaz de mantenerse a sí misma y en la cual el micobionte es el exhabitante (4). Para Ahmadjlan "Los líquenes son asociaciones fotosintéticas consistentes de apretadas poblaciones de algas verdes o cianofíceas que poseen una única combinación de caracteres, siendo primariamente fungal pero también algal" (2). Los líquenes han sido utilizados en la medicina popular por sus propiedades antibióticas, sobre todo aquellos líquenes que producen ácido úsnico (5-9), también son utilizados como alimentos Cetraria islandica, Umbilicaria sp. y Lecanora esculenta considerado el maná Hebreo, además con propósitos industriales, como productores de colorantes, en perfumería y en decoración. En la actualidad se utilizan como indicadores de contaminación ambiental, (10-11) ya que en lugares con elevada polución, son los primeros organismos que desaparecen, son muy suceptibles y registran rápidamente las variaciones de los caracteres físicos y químicos del ambiente. Existen unas pocas familias que pueden permanecer en lugares contaminados.

El micobionte fabrica sustancias que se encuentran genéricamente en los hongos, si bien la asociación con el alga les permite especificidades en las rutas biogenéticas que abren la puerta hacía las llamadas sustancias liquénicas. Se trata de productos del metabolismo secundario que se depositan en el talo (12) Los líquenes acumulan alta concentración de metabolitos que pueden almacenar en su talo, muchas de las cuales están involucradas en su actividad antimicrobiana. Entre los metabolitos liquénicos que se presentan en estos organismos están aminoácidos, azúcares, ácidos grasos, lactonas macrocíclicas, aromáticos monocíclicos, quinonas, cromonas, xantonas, terpenoides, esteroides y carotenoides. Entre los metabolitos hasta ahora aceptados como típicamente liquénicos se encuentran solamente dépsidos, depsidonas, depsonas, dibenzofuranos y ácidos úsnicos, que también se encuentran en hongos. La actividad farmacológica conocida de las sustancias liquénicas puede clasificarse en actividad antibiótica, antitumoral-mutagénica, inhibidora del virus de inmunodeficiencia humana (VIH) inhibidora enzimática y, finalmente, actividad analgésica y antipirética.

\section{Materiales y métodos}

\section{Procedencia del material botánico}

Los líquenes fueron recolectados en el margen occidental del rio Bogotá, cuenca alta ( $\mathrm{N} 05^{\circ} 07^{\prime}$ 23,4” W $73^{\circ} 39^{\prime} 10,5$ ”), un ejemplar de cada uno fue llevado a la Curadora del Herbario Forestal UDBC-Sección Criptógamas, Grupo Colombiano de Liquenología GCOL, Universidad Distrital Francisco José de Caldas y fueron determinados como: Everniastrum columbiense, Flavopunctelia flaventor, Parmotrema simulans, Ramalina celastri, Teloschistes exilis y Usnea sp,

\section{Obtención de los extractos y fracciones}

El material fue secado a temperatura ambiente durante doce días, se pulverizaron en molino hasta un tamaño de partícula adecuada para los procesos de extracción. Se tomó $100 \mathrm{~g}$ de líquenes y mediante el extractor "Soxhlet", se le realizaron 3 extracciones con disolventes de polaridad creciente: diclorometano, acetona y metanol, y también se 
les realizo extracción etanólica total, los cuales se concentraron a presión reducida en un rotavaporador y dejando evaporar a sequedad a temperatura ambiente, obteniéndose los extractos principales $\mathrm{CH}_{2} \mathrm{Cl}_{2},\left(\mathrm{CH}_{3}\right)_{2} \mathrm{CO}, \mathrm{MeOH}$ y EtOH.

\section{Evaluación de la actividad antimicrobial}

\section{Microorganismos de ensayo}

Los microorganismos se seleccionaron teniendo en cuenta su facilidad de desarrollo en medios de cultivo convencionales, su empleo en ensayos de susceptibilidad a antibióticos y su importancia médica, se utilizaron los siguientes microorganismos de prueba: Staphylococcus aureus ATCC \# 25923 y Klebsiella pneumoniae ATCC \# 70063

\section{Preparación de los inóculos}

En todo el estudio se partió de cepas de mantenimiento conservadas bajo refrigeración a $4^{\circ} \mathrm{C}$ en tubos en cuña con Agar Mueller Hinton (AMH) para bacterias. Cada una de las cepas de mantenimiento se sembró por estrías en placas con $(\mathrm{AMH})$ y se incubaron a $37^{\circ} \mathrm{C}$ por 24 horas para bacterias, al cabo de las cuales se tomó una colonia y se sembró en $5 \mathrm{ml}$ de caldo Mueller Hinton. Se incubo a $37^{\circ} \mathrm{C}$ por 18 horas, se realizaron resiembras sucesivas en el mismo caldo para asegurar que los microorganismos se encontraban en fase de crecimiento exponencial. Cada uno de estos cultivos se diluyó con solución salina estéril con el fin de obtener $10^{7}$ y $10^{8}$ UFC (Unidades Formadoras de Colonias).

\section{Método de difusión en gel por perforación en placas}

La evaluación antimicrobiana se realizó con los extractos de Flavopunctelia flaventor Extracto EtOH Total (A), Teloschistes exilis E. EtOH T. (B), Everniastrum columbiense $\mathrm{E} . \mathrm{CH}_{2} \mathrm{Cl}_{2}(\mathrm{C})$, Everniastrum columbiense E. $\left(\mathrm{CH}_{3}\right)_{2} \mathrm{CO}(\mathrm{D})$,
Everniastrum columbiense E. $\mathrm{CH}_{3} \mathrm{OH}$ (E), Parmotrema simulans $\mathrm{E}$. $\mathrm{CH}_{2} \mathrm{Cl}_{2}(\mathrm{~F})$, Parmotrema simulans E. $\left(\mathrm{CH}_{3}\right)_{2} \mathrm{CO}(\mathrm{G})$, Parmotrema simulans E. $\mathrm{CH}_{3} \mathrm{OH}(\mathrm{H})$, Usnea sp E. $\mathrm{CH}_{2} \mathrm{Cl}_{2}(\mathrm{I})$, Usnea sp E. $\left(\mathrm{CH}_{3}\right)_{2} \mathrm{CO}(\mathrm{J})$, Usnea sp E. $\mathrm{CH}_{3} \mathrm{OH}(\mathrm{K}), R a-$ malina celastri $\mathrm{E}$. $\mathrm{CH}_{2} \mathrm{Cl}_{2}(\mathrm{~L})$, Ramalina celastri $\mathrm{E}$. EtOH T. (M), Ramalina celastri E. EtOH Total $(\mathrm{N})$, mediante el método de difusión en gel por perforación en placas, el cual se describe a continuación:

En cajas de Petri con $25 \mathrm{ml}$ de $(\mathrm{AMH})$, se realizaron cuatro perforaciones equidistantes de $8 \mathrm{~mm}$ de diámetro y sello el fondo de cada una con agar. Posteriormente, se tomó un hisopo y se sumergió en la suspensión microbiana eliminando el exceso contra las paredes del tubo, y se sembró sobre la superficie del agar en tres direcciones y por el reborde de la placa, dejando secar por cinco minutos. Se agregaron las muestras para análisis; cuatro concentraciones de extractos, para ello se tomó $100 \mathrm{mg}$ de extracto seco y se diluyo en $1 \mathrm{ml}$ de DMSO, obteniéndose una dilución principal de la cual se tomaron: $(10 \mu \mathrm{l})$ correspondiente $1 \mathrm{mg}$ de extracto, $(20 \mu \mathrm{l})$ correspondiente a $2 \mathrm{mg}$, $(30 \mu \mathrm{l})$ correspondiente a $3 \mathrm{mg}$ y $(50 \mu \mathrm{l})$ correspondiente a $5 \mathrm{mg}$. Para los patrones se tomó $10 \mathrm{mg}$ de patrón (Rifaximina) y se diluyeron en $10 \mathrm{ml}$ de DMSO. La dilución principal se le realizo un factor de dilución de $1 / 2$ de la que se tomaron $(10 \mu \mathrm{l})$ correspondiente $0,005 \mathrm{mg}$ de patrón, $(20 \mu \mathrm{l})$ correspondiente $0,01 \mathrm{mg},(30 \mu \mathrm{l})$ correspondiente 0,015 mg y $(50 \mu \mathrm{l})$ correspondiente $0,025 \mathrm{mg}$. La observaciones de las respuestas antimicrobianas en estas cantidades de extracto, permitió observar una correlación entre la concentración de extracto y la acción antimicrobiana, es decir, se puede apreciar si hay una correcta difusión de las sustancias en el agar.

Una vez aplicadas las muestras se dejaron en predifusión durante 30 minutos a temperatura ambiente, se incubo a $37^{\circ} \mathrm{C}$ y se realizaron las medidas de los diámetros de inhibición a las 24 horas. 
Luego se graficó el logaritmo natural (Ln) de las masas de los extractos empleadas contra el cuadrado de los diámetros de inhibición $\left(\mathrm{X}^{2}\right)$; de esta forma se obtiene una recta que se ajusta por mínimos cuadrados y por extrapolación se halla el punto de intersección sobre la ordenada que corresponde al valor de la masa crítica (M.C.) en mg. La masa crítica se define según (12) como la masa de la sustancia antimicrobial que se difunde en la superficie del agar o gel en la interfase entre bacterias en crecimiento y bacterias inhibidas, representa una medida de la susceptibilidad de un microorganismo a prueba. Este valor se aproxima a la concentración mínima inhibitoria (CMI) obtenida en las pruebas de dilución. Se determinó la actividad antibacteriana relativa (ABR), con respecto a la Rifaximina que es equivalente a (ln C50 muestra / ln C50 rifaximina) x 100).

\section{Resultados}

Al realizar las pruebas de susceptibilidad antimicrobiana con los extractos obtenidas de líquenes se pudo determinar mediante el método de difusión en gel, que la mayoría de extractos presentaron actividad y se evidencio la presencia de halo de inhibición o disminución del crecimiento bacteriano en las diferentes concentraciones empleadas frente al grupo de bacterias Gram negativas y bacterias Gram positivas. Tablas 1 y 2 . Así mismo, siguiendo la metodología descrita, se determinó la Masa Crítica (M.C) de los extractos frente a los microorganismos de ensayo. Que representa la masa mínima de extracto para inhibir el desarrollo del microorganismo in vitro bajo las condiciones empleadas, y así mismo se demuestra que cuando las condiciones son estandarizadas se cumple que el diámetro del halo alrededor del antimicrobiano es proporcional al Ln de la concentración del mismo. Por los resultados expuestos anteriormente cabe aclarar que debido a que los extractos de líquenes están constituidos por variedad de sustancias en diferentes proporciones y diversas características, no es posible comparar la "potencia" con respecto al patrón.

Tabla 1. Actividad Antibacteriana de Staphylococcus aureus frente a Rifaximina

Staphylococcus aureus
Especie
Extracto

Ln C50

Masa crítica (mg)

ABR

\begin{tabular}{lcccc} 
Flavopunctelia flaventor & Total etanólico & 198,20 & 0,09 & 6,36 \\
\hline Teloschistes exilis & Total etanólico & 481,29 & 0,06 & 15,44 \\
\hline Everniastrum columbiense & Diclorometano & 170,96 & 0,11 & 5,49 \\
Everniastrum columbiense & Acetona & 683,86 & 0,11 & 21,94
\end{tabular}

Everniastrum columbiense

Metanólico

Parmotrema simulans

Diclorometano

Parmotrema simulans

Acetona

328,09

0,32

10,53

Parmotrema simulans

Metanólico

672,87

0,03

21,59 


\begin{tabular}{lcccc} 
Usnea sp & Diclorometano & 327,16 & 0,03 & 10,50 \\
Usnea sp & Acetona & 397,50 & 0,08 & 12,75 \\
Usnea sp & Metanolico & 118,09 & 0,91 & 3,79 \\
Ramalina celastri & Diclorometano & 98,49 & 0,98 & 3,16 \\
\hline Ramalina celastri & Total etanólico & - & - & - \\
\hline Ramalina celastri & Metanólico & 405,02 & 0,25 & 13,00 \\
\hline
\end{tabular}

Tabla 2. Actividad Antimicrobiana de Klebsiella pneumoniae frente a Rifaximina.

\begin{tabular}{|c|c|c|c|c|}
\hline \multirow{2}{*}{ Especie } & \multirow{2}{*}{ Extracto } & \multicolumn{3}{|c|}{ Klebsiella pneumoniae } \\
\hline & & Ln C50 & Masa crítica (mg) & ABR \\
\hline Flavopunctelia flaventor & Total etanólico & 334,70 & 0,26 & 8,94 \\
\hline Teloschistes exilis & Total etanólico & 835,53 & 0,03 & 22,32 \\
\hline Everniastrum columbiense & Diclorometano & 652,85 & 0,66 & 17,44 \\
\hline Everniastrum columbiense & Acetona & 560,48 & 0,02 & 14,97 \\
\hline Everniastrum columbiense & Metanólico & 514,00 & 0,23 & 13,73 \\
\hline Parmotrema simulans & Diclorometano & - & - & - \\
\hline Parmotrema simulans & Acetona & 613,23 & 0,41 & 16,38 \\
\hline Parmotrema simulans & Metanólico & 583,89 & 0,32 & 15,60 \\
\hline Usnea sp & Diclorometano & - & - & - \\
\hline Usnea sp & Acetona & 514,00 & 0,23 & 13,73 \\
\hline Usnea sp & Metanolico & - & - & - \\
\hline Ramalina celastri & Diclorometano & 267,60 & 0,95 & 7,15 \\
\hline Ramalina celastri & Total etanólico & 334,70 & 0,26 & 8,94 \\
\hline Ramalina celastri & Metanólico & 420,05 & 0,13 & 11,22 \\
\hline
\end{tabular}




\section{ABR de Extractos de liquenes respecto a rifaximina frente a Staphylococcus aureus}

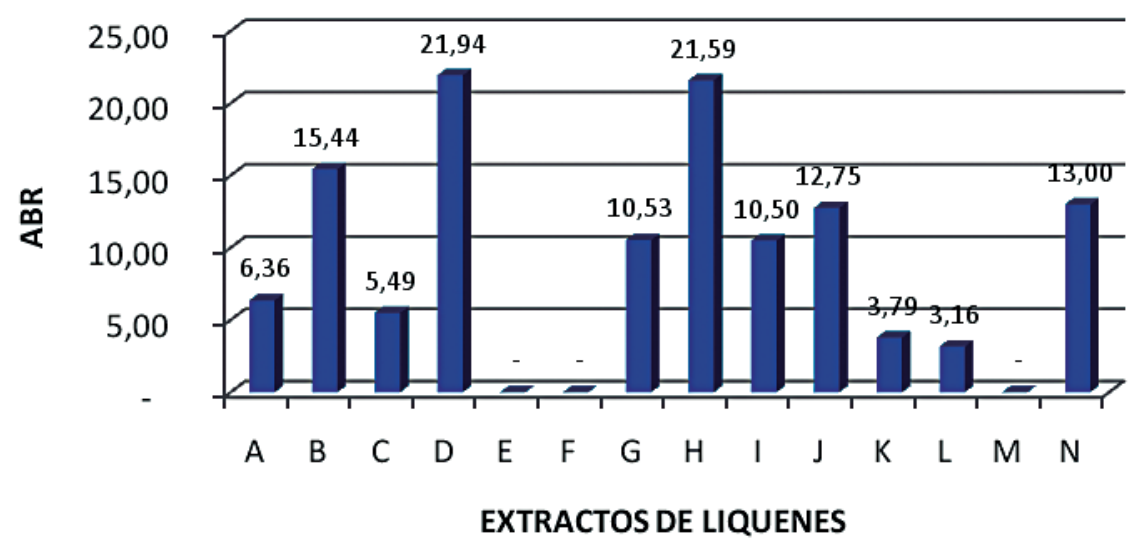

Figura 1. ABR de líquenes respecto a Rifaximina frente a Staphylococcus aureus, Flavopunctelia flaventor E. EtOH Total (A), Teloschistes exilis E. EtOH Total (B), Everniastrum columbiense E. $\mathrm{CH}_{2} \mathrm{Cl}_{2}(\mathrm{C})$, Everniastrum columbiense E. $\left(\mathrm{CH}_{3}\right)_{2} \mathrm{CO}(\mathrm{D})$, Everniastrum columbiense E. $\mathrm{CH}_{3} \mathrm{OH}(\mathrm{E})$, Parmotrema simulans E. $\mathrm{CH}_{2} \mathrm{Cl}_{2}(\mathrm{~F})$, Parmotrema simulans E. $\left(\mathrm{CH}_{3}\right)_{2} \mathrm{CO}(\mathrm{G})$, Parmotrema simulans $\mathrm{E}$. $\mathrm{CH}_{3} \mathrm{OH}$ $(\mathrm{H})$, Usnea sp E. $\mathrm{CH}_{2} \mathrm{Cl}_{2}(\mathrm{I})$, Usnea sp $\mathrm{E}$. $\left(\mathrm{CH}_{3}\right)_{2} \mathrm{CO}(\mathrm{J})$, Usnea sp E. $\mathrm{CH}_{3} \mathrm{OH}(\mathrm{K})$, Ramalina celastri $\mathrm{E}$. $\mathrm{CH}_{2} \mathrm{Cl}_{2}(\mathrm{~L})$, Ramalina celastri E. EtOH Total (M), Ramalina celastri E. EtOH Total (N).

\section{ABR de Extractos de liquenes respecto a Rifaximina frente a klebsiella pneumoniae}

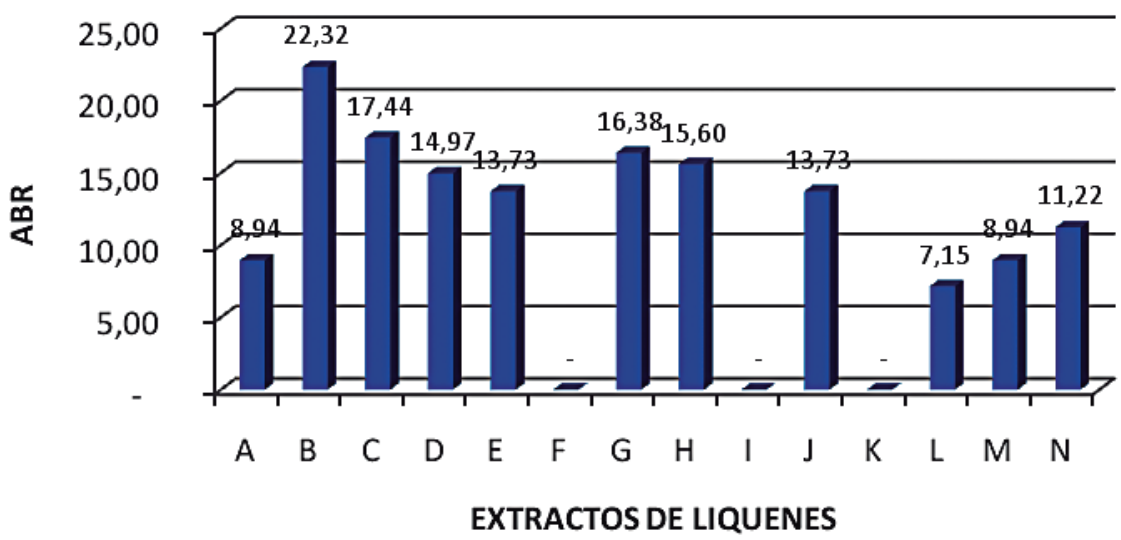

Figura 2. ABR de líquenes respecto a Rifaximina frente a Klebsiella pneumoniae, Flavopunctelia flaventor $\mathrm{E}$. EtOH Total (A), Teloschistes exilis $\mathrm{E}$. EtOH Total (B), Everniastrum columbiense $\mathrm{E}$. $\mathrm{CH}_{2} \mathrm{Cl}_{2}$ (C), Everniastrum columbiense E. $\left(\mathrm{CH}_{3}\right)_{2} \mathrm{CO}(\mathrm{D})$, Everniastrum columbiense $\mathrm{E}_{\mathrm{C}} \mathrm{CH}_{3} \mathrm{OH}(\mathrm{E})$, Parmotrema simulans $\mathrm{E} . \mathrm{CH}_{2} \mathrm{Cl}_{2}(\mathrm{~F})$, Parmotrema simulans $\mathrm{E} .\left(\mathrm{CH}_{3}\right)_{2} \mathrm{CO}(\mathrm{G})$, Parmotrema simulans $\mathrm{E}$. $\mathrm{CH}_{3} \mathrm{OH}(\mathrm{H})$, Usnea sp E. $\mathrm{CH}_{2} \mathrm{Cl}_{2}(\mathrm{I})$, Usnea sp E. $\left(\mathrm{CH}_{3}\right)_{2} \mathrm{CO}(\mathrm{J})$, Usnea sp E. $\mathrm{CH}_{3} \mathrm{OH}(\mathrm{K})$, Ramalina celastri E. $\mathrm{CH}_{2} \mathrm{Cl}_{2}(\mathrm{~L})$, Ramalina celastri E. EtOH Total(M), Ramalina celastri E. EtOH Total (N). 


\section{Discusión}

La mayoría de los microorganismos constituyen flora normal y no representan para el individuo sano normal un peligro para su salud. Sin embargo, la ruptura de ese equilibrio puede provocar el desarrollo de enfermedades infecciosas. Por ello es que se está en la búsqueda de nuevos antibióticos que provengan de productos naturales, de este estudio se puede concluir que los extractos de las especies líquenicas (Everniastrum columbiense, Flavopunctelia flaventor, Parmotrema simulans, Ramalina celastri, Teloschistes exilis y Usnea sp.), presentan actividad antibacteriana frente a los microorganismos Staphylococcus aureus (microrganismo Gram positivo), Klebsiella pneumoniae Gram (microorganismo Gram negativo) mediante el método de difusión en gel por perforación en placa.

Los extractos de Flavopunctelia flaventor (Ex. Etanólico total), Everniastrum columbiense (Ex. (Ex. Diclorometano), Parmotrema simulans (Ex. etanólico), Usnea Sp (Ex. Acetona) y Ramalina celastri (Ex. Diclorometano y Ex. Metanolico), presentaron actividad antibacteriana frente a Staphylococcus aureus con una actividad antibacteriana relativa $(\mathrm{ABR})$ con respecto a la rifaximina de 8.94, $5.49,10.53,12.75,3.16$ y 13.00 respectivamente.

Los extractos de Flavopunctelia flaventor (Ex. Etanólico total), Usnea Sp (Ex. Acetona) y $R a$ malina celastri (Ex.Diclorometano, Ex. Etanólico total y Ex. Metanólico) presentaron actividad antibacteriana frente a Klebsiella pneumoniae con una actividad antibacteriana relativa (ABR) con respecto a la rifaximina de 8.94, 13.73, 7.15, 8.94 y 11.22 respectivamente.

Como los extractos de diferentes polaridades de líquenes están constituidos por variedad de sustancias en diferentes proporciones y diversas características, no es posible comparar la "potencia" con respecto al patrón. Por ello amerita purificar e identificación los extractos que presentaron actividad frente a bacterias Gram positvas y Gram negativas. Y así verificar el o los metabolitos secundarios que presentan dicha actividad.

Teniendo en cuenta los resultados, vemos que los líquenes Flavopunctelia flaventor Everniastrum columbiense, Parmotrema simulans, Ramalina celastri, Teloschistes exilis y Usnea sp. Son especies promisorias y por tanto es importante realizar otros estudios como son actividad citotóxica, actividad antioxidante, actividad antiinflamatoria y actividad antiparasitaria.

El trabajo de Gómez, et al (27), mediante estudios físico químicos y microbiológicos informan que muchos de los órganos con estas afecciones son aptos para el consumo humano y no deberían generar pérdida por la retención del producto en las plantas de sacrificio, allí se recomendó evaluar la integridad de la totalidad de los tejidos de los miembros plantares. En respuesta, este estudio obtiene evidencias morfológicas para el $78 \%$ de los casos evaluados que pueden apoyar los análisis del estudio anterior.

De la misma forma, solo en el $22 \%$ de las lesiones evaluadas el aspecto macroscópico y las lesiones microscópicas de las piezas podrían apoyar la decisión de retención por parte de los inspectores, lo cual sugiere discutir, con las entidades de control y vigilancia del Estado, pautas más claras que definan las causas técnicas para la retención de piezas a nivel de planta de sacrificio.

\section{Agradecimientos}

Esta investigación fue financiada por la VicerrecA la facultad de Ingeniería Ambiental de la Universidad El Bosque. 


\section{Referencias}

1. Sipman, H. \& J. Aguirre, C. Contribución al conocimiento de los Líquenes de Colombia I. Clave genérica para los líquenes foliosos y fruticosos de los páramos colombianos. Caldasia, 1982;13 (64): 603-634.

2. Ahmadjian, V.; M.E. Hale, eds. The Lichens. New York. Academic Press, 1973,697 pp. Lichens are more important than you think, BioScience 1995; 45 (3): 124

3. Filho, LX; CT Rizzini. Manual de liquenologia brasileiro. Ed. Universidade Federal de Pernambuco. 1973, 431 pp.

4. Hawsworth, D. L.. Interacciones Hongo - Alga en simbiosis liquènicas y liquenoides. Anales Jardín Botánico de Madrid. 1989; 46 (1):235-247.

5. Mahadik N., Behera B., Makhija U., Morey M. Actividad protectora, antioxidantes y - Cardiovasculares actividades antimicrobianas de un liquen especie Usnea complanata, Nueva Biotecnología. 2009; 25(1): 58

6. Gulluce M., Aslan A., Sokmen M., Sahin F., Adiguzel A. Agar G, Sokmen A. La detección de los antioxidantes y antimicrobianos propiedades del líquenes Parmelia saxatilis, Platismatia glauca, Ramalina polinarios, polymorpha Ramalina y Umbilicaria nylanderiana, Phytomedicine. 2006; 13 (7): 515-521.

7. Celenza Giuseppe, Segatore Bernardetta, Setacci Domenico, Bellio Pierangelo , Brisdelli Fabrizia, Piovano Marisa, Garbarino Juan A., Nicoletti Marcello, Perilli Mariagrazia, Amicosante Gianfranco , In vitro la actividad antimicrobiana de pannarin solo y en combinación con los antibióticos contra la meticilina-esistente Staphylococcus aureus aislamientos clínicos , Phytomedicine . 2012; 19 (7): 596-602.
8. Manojlović Nedeljko, Ranković Branislav, Kosanić Marijana, Vasiljević Perica, Stanojković Tatjana, Composición química de tres Parmelia líquenes y antioxidante, antimicrobiana y citotóxicos actividades de algunos de sus principales metabolitos, Fitomedicina. 2012; 19 (13): 1166-1172.

9. Kosanić Marijana, Manojlović Nedeljko, Jankovic Slobodan, Stanojković Tatjana, Ranković Branislav, Evernia prunastri y Pseudoevernia furfuraceae líquenes y sus principales metabolitos como antioxidante, antimicrobiano y agentes anticancerígenos, Food and Chemical Toxicology. 2013; 53:112118.

10. Rubiano L.J. Líquenes como indicadores de contaminación en la Termoeléctrica de Zipaquirá y el Complejo Industrial de Betania, Cund. Acta biol Colomb. 1989;4:95-125.

11. Rubiano LJ. Delimitación de áreas de isocontaminación en Cali y Medellín utilizando líquenes como indicadores. PérezArbelaezia. 1987;1(4-5):7-41.

12. Toledo, F., A. García, F. León \& J. Bermejo., Ecología química en hongos y líquenes. Rev.Acad. Colomb. Cienc. 2004,28 (109): 509-528. 